\title{
Surpassing Rayleigh limit: Fisher information analysis of partially coherent source(s)
}

Lee, Kwan Kit, Ashok, Amit

Kwan Kit Lee, Amit Ashok, "Surpassing Rayleigh limit: Fisher information analysis of partially coherent source(s)," Proc. SPIE 11136, Optics and Photonics for Information Processing XIII, 111360H (6 September 2019); doi: $10.1117 / 12.2528540$

EDIE Event: SPIE Optical Engineering + Applications, 2019, San Diego, California, United States 


\title{
Surpassing Rayleigh Limit: Fisher information analysis of partially coherent source(s)
}

\author{
Kwan Kit Lee ${ }^{\mathrm{a}}$ and Amit Ashok ${ }^{\mathrm{a}, \mathrm{b}}$ \\ a1630 E University Blvd, College of Optical Sciences, \\ University of Arizona, Tucson, AZ, USA, 85721 \\ ${ }^{\mathrm{b}} 1230$ E Speedway Blvd, Department of Electrical and Computer Engineering, \\ University of Arizona, Tucson, AZ, USA, 85721
}

\begin{abstract}
Tsang et al. have shown that the Fisher information of the two incoherent point source separation, below the Rayleigh limit, is finite and achievable using optical modes measurements. ${ }^{1}$ However, recent claims regarding partial coherence of sources, no matter how small, leads to necessarily zero Fisher information as the source separation decreases below the Rayleigh limit approaching zero have proved to be controversial. ${ }^{2,3}$ Thus, the impact of partial coherence on the photon counting optical modal measurements merits further exploration. In this work, we derive the mutual coherence function (image plane) of two partially coherent point sources and find the classical Fisher information of the source separation using both direct image plane and photon counting modal measurements. A classical Fisher information analysis of partially coherent source(s) leads to some rather surprising results for two-point source resolution as the source separation approaches zero. We find that the magnitude of the Fisher information strongly depends on the degree of (positive/negative) partial coherence, which can be understood using an intuitive semi-classical analysis of direct image plane and photon counting modal measurements. We also provide an error analysis of the maximum likelihood estimators for both measurements.
\end{abstract}

Keywords: Fisher information, coherent point sources, Rayleigh limit

\section{INTRODUCTION}

In traditional imaging, the Rayleigh's resolution criteria typically defines the resolving limit of an optical imaging system. ${ }^{4}$ The criterion states that in order to distinguish two points in the image plane, the separation of the two points has to be larger than the width of the point spread function (PSF) of the system, which is ultimately limited by diffraction. However, more rigorously the ultimate resolving (i.e. angular separation) accuracy for two point-sources is determined by the Fisher information of the image measurement. ${ }^{5}$ It can be shown that this Fisher information approaches zero as the angular separation of the two point source converges, below the diffraction limit, to zero. However, quantum information theory has shown that two close incoherent point sources can be resolved by using linear optics and photon counting measurements even when their separation is smaller than Rayleigh's criterion. ${ }^{1}$ It has been demonstrated that by counting the photons in terms of the various spatial modes of the optical field, both classical and quantum Fisher Information approach finite values when the two point source separation goes to zero. This result implies that a finite precision can be achieved in the deep sub-Rayleigh regime, which is not achievable by the traditional direct image plane measurement.

Recently, the classical Fisher information and the quantum Fisher information for two partially coherent point sources has been also being investigated. ${ }^{2}$ This work shows that if there is any finite degree of coherence between the two-point sources, no matter how small, the finite precision in the limit two point separation approaches zero would fade (i.e. Rayleigh's curse), except for perfectly positively-correlated sources. Counterarguments, ${ }^{3}$

Further author information: (Send correspondence to Prof. Amit Ashok)

Kwan Kit Lee (E-mail): kklee@email.arizona.edu

Amit Ashok (E-mail): ashoka@optics.arizona.edu

Optics and Photonics for Information Processing XIII, edited by Khan M. Iftekharuddin,

Abdul A.S. Awwal, Victor H. Diaz-Ramirez, Andrés Márquez, Proc. of SPIE Vol. 11136

$111360 \mathrm{H} \cdot$ @ $2019 \mathrm{SPIE} \cdot \mathrm{CCC}$ code: 0277-786X/19/\$21 · doi: 10.1117/12.2528540 
which have debated the parametrization of the correlation function and the normalization of the density operator originally reported in Ref. 2, have been made and some of these points were conceded by the authors. ${ }^{6}$ However, the normalization issue in this analysis has still proven to be controversial. In this work, we adopt a semi-classical approach to the analysis of partially coherent two-point sources and find the classical Fisher information of their angular separation using both imaging and photon counting measurements. We demonstrate that even for partially-coherent sources the Rayleigh's curse is not manifested, except perfectly positively coherent sources. We validate our Fisher information analysis by quantifying the precision of the angular separation estimate for various degrees of coherence and separation of two point sources.

\section{PHOTON DISTRIBUTION: DIRECT IMAGING AND MODE SORTING MEASUREMENTS}

\subsection{Propagation of Mutual Coherence}

We begin by defining the mutual intensity of the two point sources denoted by $J_{o}\left(x_{o 1}, x_{o 2} ; \theta\right)$ :

$$
\begin{aligned}
J_{o}\left(x_{o 1}, x_{o 2} ; \theta\right)= & {\left[I\left(x_{o 1} ; \theta\right) I\left(x_{o 2} ; \theta\right)\right]^{1 / 2} \mu_{o}\left(x_{o 1}-x_{o 2}\right) } \\
= & \frac{I_{0} \mu_{o}\left(x_{o 1}-x_{o 2}\right)}{2}\left[\delta\left(x_{o 1}-\frac{\theta}{2}\right) \delta\left(x_{o 2}-\frac{\theta}{2}\right)+\delta\left(x_{o 1}+\frac{\theta}{2}\right) \delta\left(x_{o 2}-\frac{\theta}{2}\right)\right. \\
& \left.+\delta\left(x_{o 1}-\frac{\theta}{2}\right) \delta\left(x_{o 2}+\frac{\theta}{2}\right)+\delta\left(x_{o 1}+\frac{\theta}{2}\right) \delta\left(x_{o 2}+\frac{\theta}{2}\right)\right]^{1 / 2}
\end{aligned}
$$

where $x_{o 1}, x_{o 2}$ are the angular co-ordinates in the object plane and $\theta$ is the angular separation of two point source as shown in Fig. 1. The complex degree of coherence, denoted by $\mu\left(x_{o 1}-x_{o 2}\right)$, describes the coherence of the two point sources. ${ }^{7}$ We consider a simple imaging system with a single lens (Fig. 1). After propagating to the image plane, under paraxial condition and assuming Gaussian aperture, the mutual coherence can be written as: ${ }^{7}$

$$
\begin{aligned}
J\left(x_{1}, x_{2} ; \theta\right)= & \frac{\bar{n}}{2 \sqrt{2 \pi} \sigma_{i}}\left\{\exp \left[\frac{1}{4 \sigma_{i}^{2}}\left(\left(x_{1}-\theta / 2\right)^{2}+\left(x_{2}-\theta / 2\right)^{2}\right)\right]\right. \\
& +\exp \left[\frac{1}{4 \sigma_{i}^{2}}\left(\left(x_{1}+\theta / 2\right)^{2}+\left(x_{2}+\theta / 2\right)^{2}\right)\right] \\
& +\mu(\theta) \exp \left[\frac{1}{4 \sigma_{i}^{2}}\left(\left(x_{1}-\theta / 2\right)^{2}+\left(x_{2}+\theta / 2\right)^{2}\right)\right] \\
& \left.+\mu^{*}(\theta) \exp \left[\frac{1}{4 \sigma_{i}^{2}}\left(\left(x_{1}+\theta / 2\right)^{2}+\left(x_{2}-\theta / 2\right)^{2}\right)\right]\right\},
\end{aligned}
$$

where $x_{1}, x_{2}$ are co-ordinates of any two points on the optical field the image plane and $\bar{n}$ is proportional to the photons emitted from the two point sources. The width of the Gaussian PSF is given by: $\sigma_{i}=\lambda / 4 \pi \sigma_{a}$, where $\lambda$ is the wavelength and $\sigma_{a}$ is the standard deviation of the Gaussian function describing the aperture. We summarize the pre-factor of Eq. (2) by $\bar{n}$, which is proportional to the intensity $I_{o}$.

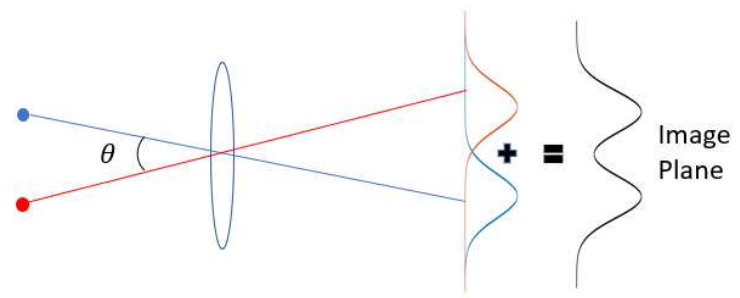

(a) Direct imaging measurement.

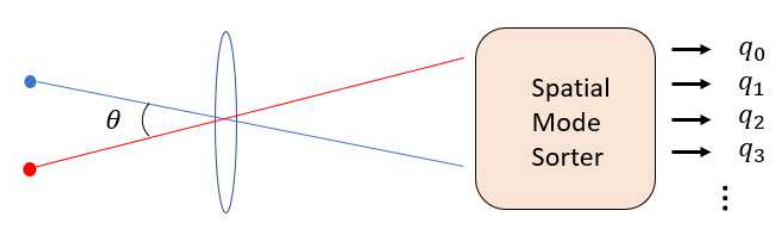

(b) Mode sorting measurement.

Figure 1: The system schematic illustrating the two measurements. 


\subsection{Direct Imaging Model}

Consider pixellated detectors in the image plane as shown in Fig. 1(a). By setting $x_{1}=x_{2}=x$ in Eq. (2), the intensity in the image plane can be written as:

$$
I(x ; \theta)=\frac{\bar{n}}{2 \sqrt{2 \pi} \sigma_{i}}\left\{\exp \left[-\frac{(x-\theta / 2)^{2}}{2 \sigma_{i}^{2}}\right]+\exp \left[-\frac{(x+\theta / 2)^{2}}{2 \sigma_{i}^{2}}\right]+2 \operatorname{Re}[\mu(\theta)] \exp \left[-\frac{x^{2}+(\theta / 2)^{2}}{2 \sigma_{i}^{2}}\right]\right\} .
$$

Note that Eq. (3) is normalized such that $I(x ; \theta, \mu)=1$ when $\bar{n}=1$ and $\mu(\theta)=0$ (incoherence). The intensity, which is assumed to be pseudo continuous on the detector plane, is the mean number of photons collected as described by the Poisson point process on the pixellated detectors.

\subsection{Mode sorting Measurement Model}

Fig. 1(b) shows the schematic illustrating the mode sorting measurement. Spatial mode demultiplexer (SPADE) is used to sort the optical field incident in the image plane into a spatial mode basis. Photons in each of the different spatial modes are counted. In order to find the optical field intensity in each mode, we have to project the mutual coherence function onto mode basis. If we use Hermite Gaussian mode set as the mode basis, ${ }^{1,2}$ the mean number of photon in the $q^{t h}$ mode $\psi_{q}(x)$, labelled by $\lambda_{q}(\theta)$, can be written as:

$$
\begin{aligned}
\lambda_{q}(\theta) & =\iint_{-\infty}^{\infty} \psi_{q}\left(x_{2}\right) J\left(x_{1}, x_{2} ; \theta\right) \psi_{q}\left(x_{1}\right) d x_{1} d x_{2} \\
& =\frac{\bar{n}}{q !}\left\{1+(-1)^{q} \operatorname{Re}[\mu(\theta)]\right\}\left(\frac{\theta^{2}}{16 \sigma_{i}^{2}}\right)^{q} \exp \left(-\frac{\theta^{2}}{16 \sigma_{i}^{2}}\right) .
\end{aligned}
$$

Strictly speaking, one should integrate Eq. (4) across the detector size. However, if $\theta$ is small enough such that $J\left(x_{1}, x_{2} ; \theta\right) \ll 1$ when $x_{1}$ or $x_{2}$ is large enough, we may approximate the integral as in Eq. (4) to obtain the analytical form.

\section{MEASUREMENT PERFORMANCE METRICS: FISHER INFORMATION AND MEAN SQUARE ERROR}

For each measurement described in Section 2, the classical Fisher information quantifies the information relevant to estimating the two point source separation and can be expressed as:

$$
\begin{aligned}
& \mathrm{FI}_{\text {imag }}(\theta)=\int_{-\infty}^{\infty} \frac{1}{I(x ; \theta)}\left[\frac{\partial I(x ; \theta)}{\partial \theta}\right]^{2} d x \\
& \mathrm{FI}_{\text {mode }}(\theta)=\sum_{q=0}^{\infty} \frac{1}{\lambda_{q}(\theta)}\left[\frac{\partial \lambda_{q}(\theta)}{\partial \theta}\right]^{2} .
\end{aligned}
$$

Up to now, the coherence $\mu(\theta)$ is expressed as a function of $\theta$. In our analysis, we assume that it is a constant and calculate the maximum likelihood estimator (MLE) for some representative values of $\mu$. It is worth noting that the MLE approaches a minimum variance unbiased estimator as $\bar{n}$ and $\theta$ get large, so we use it here to obtain MSE to quantify estimation performance of the two measurements. Here, for the two-point source problem, given a measurement $\vec{n}=\left(n_{1}, n_{2}, n_{3} \ldots\right)$, the MLE $\hat{\theta}$ can be numerically computed. We estimate the MSE of the two measurements by Monte Carlo sampling:

$$
\operatorname{MSE} \approx<\left(\theta-\hat{\theta}_{m}\right)^{2}>
$$

where $\hat{\theta}_{m}$ is ML estimation of each sample measurement. 


\section{ANALYSIS OF FISHER INFORMATION AND MLE}

We demonstrate the theory formulated above with some representative cases. Here the angular separation of the object ranges from 0 to $6 \times 10^{-5}$ radians. $\lambda$ is set to be $500 \mathrm{~nm}$ and $\sigma_{a}$ is set to be $15000 \lambda$. $\sigma_{i}$ is the width of the Gaussian PSF on the image plane. The source coherence $\mu$ is chosen to be $0, \pm 0.1$, and \pm 0.9 , which represent incoherent and strongly/weakly positively/negatively correlated sources. For the analysis in this section, we assume that the measurement exposure time window is fixed. Rayleigh limit is typically defined for a circular aperture. Here for a Gaussian aperture we use full width at half maximum (FWHM) $\sigma_{F}$ of the PSF as the Rayleigh limit $\left(\sigma_{F}=2.36 \sigma_{i}\right)$.

\subsection{Fisher Information Analysis}

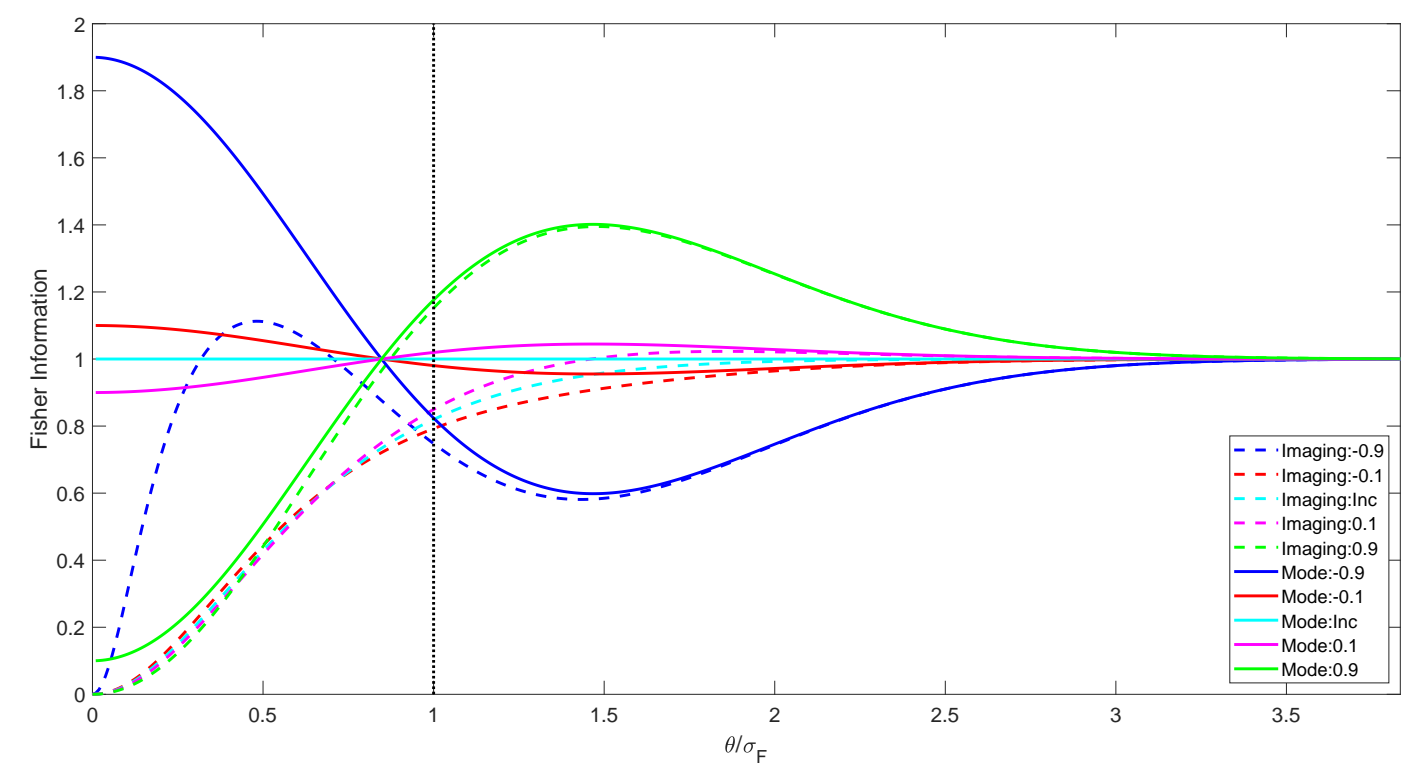

Figure 2: Fisher information for imaging (dashed) and mode sorting (solid) measurements for varying degrees (colors) of source coherence. The black dotted line shows the FWHM (Rayleigh's limit).

Fisher information for different degrees of coherence are shown in Fig. 2. For the mode sorting measurement, we note that the Fisher information attains finite values when the separation of the two point source approaches zero. The Fisher information for incoherent sources is a constant. ${ }^{3}$ If the number of photon received is fixed (i.e. variable exposure time), the quantum Fisher information has the similar properties qualitatively except for $\mu=-1 .{ }^{6}$ For direct imaging, all the Fisher information goes to zero as $\theta$ goes to zero for all coherence values.

Here there are some FI trends worth highlighting. First, the Fisher information of mode sorting measurement is always higher than that of the imaging measurement, which implies that the best unbiased estimator of the mode sorting measurement always outperforms that of the direct imaging measurement. Second, in the small $\theta$ regime, more negatively correlated sources lead to higher Fisher information than positively correlated sources. This can be understood intuitively: negative correlation gives rise to a minima (null) on the optical axis, as shown in Fig. 2, at $x=0$ when $\theta$ is small while positive correlation does the opposite. The dip (or the minima) surrounded by maximas on either side encodes more information and thus facilitates more accurate estimation of the unknown parameter $\theta$.

\subsection{Maximum Likelihood Estimation: Error analysis}

We quantify the performance of the maximum likelihood estimator (MLE) by estimating its mean square error (MSE). Simulations of $n_{i}$, the number of photons found in each mode/pixel following the Poisson distribution 


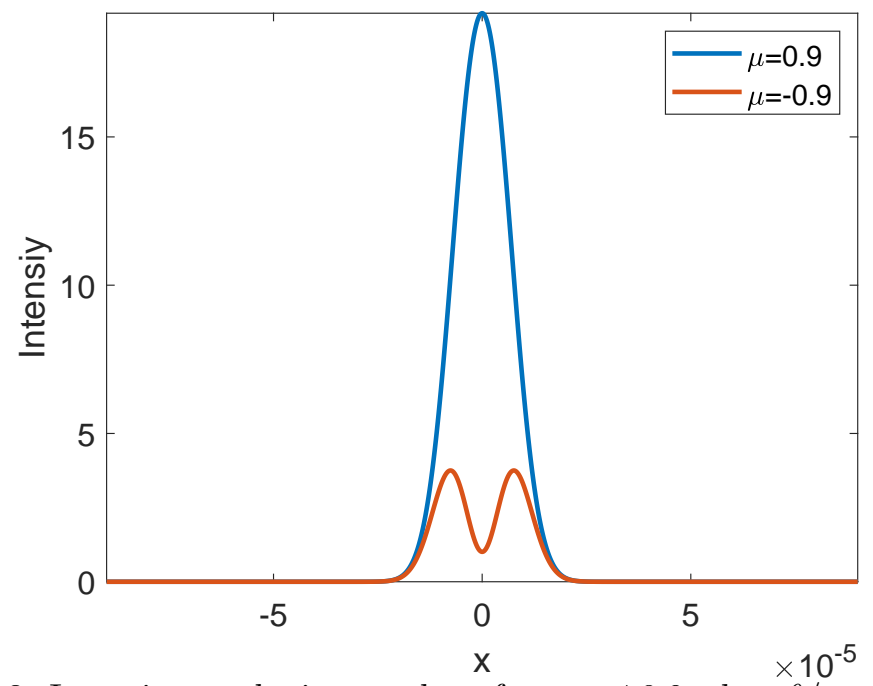

Figure 3: Intensity on the image plane for $\mu= \pm 0.9$ when $\theta / \sigma_{F} \approx 0.68$.

with the mean shown in Eqs. (3) and (4), are carried out for $\bar{n}=10$ and $\bar{n}=100$. MSEs are estimated by Eq. $=(7)$. The results, together with the Cramer-Rao lower bound (CRLB) are shown in Fig. 4. CRLB, lower bound on MSE, is obtained as the inverse of the Fisher information defined in Eqs. (5) and (6). Note that all the bounds converge to $1 / \bar{n}$.

For Fig. 4, it can be seen that the MSE of mode sorting tends to zero for negative and small positive coherence $(\mu)$. This behavior can be explained as follow. In order to find the MSE at $\theta=0$, one has to solve the following equation:

$$
\theta^{2}\left[n_{t o t}-2 \mu \bar{n} \exp \left(-\frac{\theta^{2}}{8 \sigma_{i}^{2}}\right)\right]=0,
$$

where $n_{t o t}$ denotes the total number of photon received in the finite exposure window. The solution for Eq. (8) has qualitative difference when $\mu$ varies:

$$
\hat{\theta}= \begin{cases}0 & , \text { if } \mu \leq 0 \text { or } n_{t o t}>2 \mu \bar{n} . \\ \sigma_{i} \sqrt{8 \ln \left(\frac{2 \mu \bar{n}}{n_{t o t}}\right)} & , \text { otherwise. }\end{cases}
$$

At $\theta=0$, one can only find photons in the $0^{t h}$-order mode, under which the MLE predicts $\theta=0$ when $\mu \leq 0$ according to Eq. (9). If $\mu>0$, the larger the $\mu$ is, the lower probability that the estimator gives the correct prediction. Although the MSE for the imaging measurement does not goes to zero as $\theta$ goes to zero, it still has finite value at $\theta=0$.

Furthermore, if one has the exact knowledge of the actual total number of photon received, the MSE, no matter what the number, converges to the corresponding CRLB. However, if we just have the knowledge of the average or expected number of photons, the MSE can only converge to the weighted average of the CRLB for different number of photons. Thus when $\bar{n}$ is large, the MSE converges to CRLB asymptotically. 


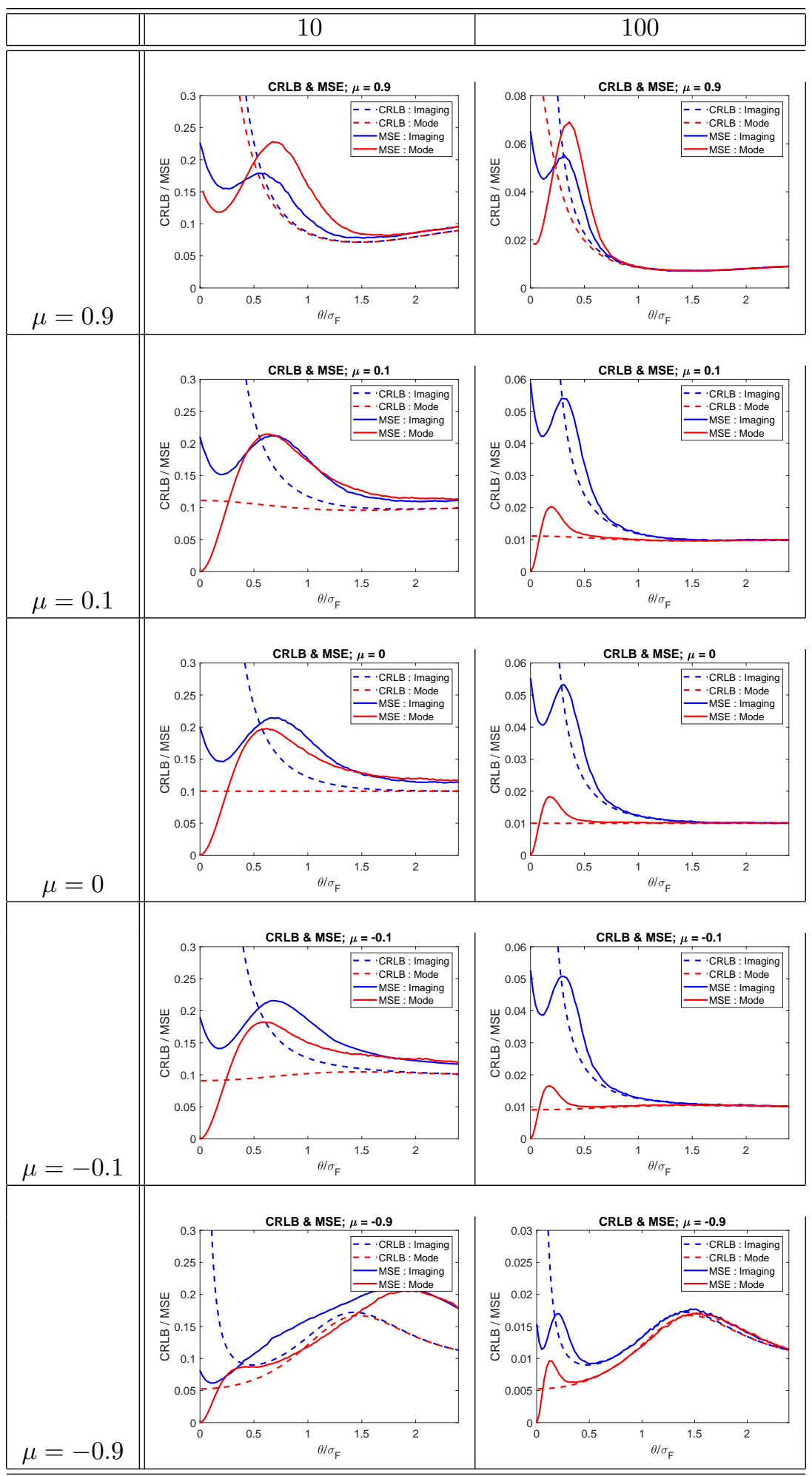

Figure 4: MSE and CRLB for the two measurements. 


\section{CONCLUSION}

In this work we employ mutual coherence analysis to determine the photon distribution on the image plane for both imaging and mode sorting measurement for the task of resolving two (partially) coherent point source. We find that two perfectly coherent point sources still lead to finite Fisher information if mode sorting measurement is used. We also validated our Fisher information analysis with evaluating the achievable MSE (i.e. separation precision) for both types of measurements.

\section{ACKNOWLEDGEMENT}

Authors gratefully acknowledge support from Defense Advanced Research Projects Agency (DARPA) REVEAL program under contract number HR0011-16-C-0026.

\section{REFERENCES}

[1] Tsang, M., Nair, R., and Lu, X.-M., "Quantum theory of superresolution for two incoherent optical point sources," Phys. Rev. X 6, 031033 (Aug 2016).

[2] Larson, W. and Saleh, B. E. A., "Resurgence of rayleigh's curse in the presence of partial coherence," Optica 5, 1382-1389 (Nov 2018).

[3] Tsang, M. and Nair, R., "Resurgence of rayleigh's curse in the presence of partial coherence: comment," Optica 6, 400-401 (Apr 2019).

[4] Born, M., Born, L., Wolf, E., BORN, M., Bhatia, A., Clemmow, P., Gabor, D., Stokes, A., Taylor, A., Wayman, P., et al., [Principles of Optics: Electromagnetic Theory of Propagation, Interference and Diffraction of Light], Cambridge University Press (1999).

[5] Kay, S. M., [Fundamentals of Statistical Signal Processing: Estimation Theory], Prentice-Hall, Inc., Upper Saddle River, NJ, USA (1993).

[6] Larson, W. and Saleh, B. E. A., "Resurgence of rayleigh's curse in the presence of partial coherence: reply," Optica 6, 402-403 (Apr 2019).

[7] Goodman, J., [Statistical Optics], A Wiley-Interscience publication, Wiley (1985). 